\title{
Non-invasive predictive model for hepatic venous pressure gradient based on a 3-dimensional computed tomography volume rendering technology
}

\author{
YUJEN TSENG $^{1 *}$, LILI MA $^{2 *}$, TIANCHENG LUO $^{1}$, XIAOQING ZENG $^{1}$, NA LI $^{1}$, \\ YICHAO WEI $^{1}$, JI ZHOU ${ }^{1}$, FENG LI ${ }^{1}$ and SHIYAO CHEN ${ }^{3}$
}

Departments of ${ }^{1}$ Gastroenterology and ${ }^{2}$ Endoscopy, Zhongshan Hospital, Fudan University; ${ }^{3}$ Department of Gastroenterology, Endoscopy Center, Evidence-Based Medicine Center, Zhongshan Hospital, Fudan University, Shanghai 200032, P.R. China

Received June 3, 2017; Accepted November 17, 2017

DOI: $10.3892 /$ etm.2018.5816

\begin{abstract}
Portal hypertension secondary to liver cirrhosis may cause a number of life-threatening complications. The rupture of gastroesophageal varices is associated with a high mortality rate of $15-30 \%$. Hepatic venous pressure gradient (HVPG) is an accurate reflection of disease severity, however this can only be assessed via an invasive interventional procedure. The aim of the present study was to explore a non-invasive method based on 3D computed tomography (CT) volume rendering technology to accurately predict HVPG. A total of 77 patients diagnosed with liver cirrhosis underwent HVPG examination in the present study and the appropriate clinical and radiological data were retrospectively reviewed. A 3D liver and spleen volume rendering was constructed for volume measurements. All non-invasive parameters were tested using univariate analysis and the resulting variables that were
\end{abstract}

Correspondence to: Dr Shiyao Chen, Department of Gastroenterology, Endoscopy Center, Evidence-Based Medicine Center, Zhongshan Hospital, Fudan University, 180 Fenglin Road, Shanghai 200032, P.R. China

E-mail: chen.shiyao@zs-hospital.sh.cn

*Contributed equally

Abbreviations: HVPG, hepatic venous pressure gradient; CSPH, clinically significant portal hypertension; EGD, esophagogastroduodenoscopy; GOV, gastroesophageal varices; IGV, isolated gastric varices; EBL, endoscopic band ligation; EIS, endoscopic injection sclerotherapy; BRTO, balloon-occluded retrograde transvenous obliteration; FHVP, free hepatic venous pressure; WHVP, wedged hepatic venous pressure; CT, computed tomography; APRI, aminotransferase-to-platelet ratio index; ROC, receiver operating characteristic; AUROC, area under receiver operating characteristic; NPV, negative predictive value; PPV, positive predictive value

Key words: portal hypertension, computer assisted diagnosis, liver cirrhosis, computed tomography, volume computer tomography statistically significant $(\mathrm{P}<0.20)$ were used in the multivariate linear regression model. The HVPG predictive model was as follows: $\mathrm{HVPG}=18.726-0.324$ (albumin) +1.57 (aminotransferase-to-platelet ratio index) +0.004 (liver volume) (multivariate regression analysis, $\mathrm{P}=0.006)$. The corresponding area under receiver operating characteristic curve to identify clinically significant portal hypertension defined as $\mathrm{HVPG} \geq 10 \mathrm{mmHg}$ was 0.810 (95\% confidence interval; $0.705-0.891$ ), with an optimal cut-off value of 12.84 , yielding a sensitivity of $80.36 \%$ a specificity of $76.19 \%$. The results of the present study indicate that 3D CT volume rendering technology may have the potential to be used for non-invasive prediction of HVPG.

\section{Introduction}

Portal hypertension is a progressive complication secondary to intra-hepatic, pre-hepatic or post-hepatic etiologies (1). Liver cirrhosis is the most common intra-hepatic cause of portal hypertension and affects $\sim 1 \%$ of the worldwide population, primarily in Asia and Africa (1). Although portal hypertension is associated with a series of complications, including ascites, hepatic encephalopathy, hepatorenal syndrome and portal venous thrombosis, the most acute and common gastrointestinal emergency is gastroesophageal variceal rupture $(2,3)$. The prevalence of gastroesophageal varices is $\sim 50 \%$ among patients with cirrhosis at the time of diagnosis, with a higher prevalence amongst Child-Pugh class B and C patients (3). Rupture or gastroesophageal hemorrhage may lead to uncontrollable torrential hemorrhage, which is associated with a high mortality rate of $15-30 \%$-possibly higher in developing countries (4). The incidence of gastroesophageal varices is associated with the hepatic venous pressure gradient (HVPG) (5). HVPG is an accurate reflection of disease severity; however, measurement involves an invasive interventional procedure that is not always readily available, particularly in primary and hospitals (6). Patients with HVPG measurements $\geq 10 \mathrm{mmHg}$ are considered to have clinically significant portal hypertension (CSPH) (7). HVPG is also used to monitor prognostic and therapeutic indications. A decrease in HVPG $>20 \%$ from the baseline, or to $\leq 12 \mathrm{mmHg}$, 
is clinically substantial (5-7). According to the Baveno VI Consensus Workshop, CSPH is predictive of gastroesophageal varices and decompensation, which requires a prompt primary prophylactic treatment with either non-selective beta-blockers or endoscopic therapy (7). Various alternative non-invasive techniques have been explored, including non-invasive biomarkers, Doppler sonography, transient elastography, computed tomography $(\mathrm{CT})$ and magnetic resonance imaging, in an attempt to accurately predict HVPG and the risk of gastroesophageal variceal hemorrhage in patients with portal hypertension $(5,6,8)$. However, the assessment of portal hypertension is HVPG-driven and, at present, does not have a non-invasive equivalent (9). The aim of the present study was to explore the potential of liver and spleen volume values calculated using 3D CT-rendering technology to provide an accurate diagnosis of HVPG. The improved availability of clinically significant data would allow for prompt primary prophylaxis intervention, which may result in lower mortality and morbidity rates in patients with decompensated cirrhosis.

\section{Patients and methods}

Study population. A total of 161 patients diagnosed with cirrhosis of varying etiologies underwent HVPG examination at Zhongshan Hospital, Fudan University (Shanghai, China), a tertiary medical center, between March 2013 and August 2015. A diagnosis of cirrhosis was made according a series of physical [presence of liver palm, spider angioma, hepatic encephalopathy based on clinical symptoms, including cognitive dysfunction, asterixis and increased ammonia level levels $(18-72 \mu \mathrm{mol} / \mathrm{l})]$ (10), laboratory [complete blood count: Hemoglobin (115-150 g/l), platelet (125-350x10 $/ 1)$ liver function: total bilirubin (3.4-20.4 $\mu \mathrm{mol} / \mathrm{l})$, albumin (35-55 g/l), aspartate aminotransferase (AST; 7-40 U/l), alanine aminotransferase (ALT; 13-35 U/1) and renal function: Serum creatinine $(44-115 \mu \mathrm{mol} / \mathrm{l})$, coagulation tests: Prothrombin time (10.0-13.0 sec)], radiological (computed tomography or magnetic resonance imaging of the liver presenting as nodular hepatic contour or changes in volume distribution) or histological [liver biopsy and METAVIR score (11)] findings. The inclusion criteria were as follows: i) No previous treatment for portal hypertension or gastroesophageal varices; and ii) diagnosis of gastroesophageal varices confirmed through an endoscopic exam. A total of 44 patients were excluded from the present study due to a prior history of splenectomy. A further 34 patients were excluded due to concurrent conditions that had the potential to affect the accuracy of HVPG measurements, including 3 patients with hepatocellular carcinoma and 31 with portal venous thrombosis evident on imaging exams. A total of 6 patients had insufficient radiological data to construct a $3 \mathrm{D}$ rendering model for liver and spleen volume and so were excluded from the study cohort. The final study population comprised 77 patients and the appropriate clinical (laboratory parameters) and radiological data (CT imaging studies) were retrospectively reviewed via an unique patient identification number. Patient data are presented in Table I. The present study was approved by the Ethics Committee of Zhongshan Hospital Fudan University (approval no. B2015-133R; Shanghai, China). Prior informed consent was provided by all patients.
Endoscopic examination. All patients underwent an esophagogastroduodenoscopy (EGD) examination performed by one of two experienced endoscopists, each with over 15 years of experience. The presence of gastroesophageal varices (GOV) was assessed and graded according to Sarin's classification (12). GOV type 1 appears as a continuation of esophageal varices and extends along the lesser curvature of the stomach, whereas GOV type 2 extends beyond the gastro-esophageal junction into the fundus. IGV type 1 are located in the fundus and IGV type 2 are ectopic varices that may be located in the gastric body, antrum or pylorus $(12,13)$. Patients were treated appropriately with different endoscopic procedures based on the degree and presentation of gastroesophageal varices under endoscopic observation. Treatment methods included endoscopic band ligation (EBL), endoscopic injection sclerotherapy (EIS) for esophageal varices, cyanoacrylate injection for gastric varices, EBL combines with cyanoacrylate injection for concurrent esophageal and gastric varices and finally balloon-occluded retrograde transvenous obliteration (BRTO) for patients with gastric varices and spontaneous portosystemic shunts.

$H V P G$. Patients were placed in a supine position and given local percutaneous anesthesia ( $0.1 \mathrm{~g}$ lidocaine hydrochloride injection; Shandong Hualu Pharmaceutical Co., Ltd., Liaocheng, China). A balloon catheter (Synergy; Boston Scientific, Boston, MA, USA) attached to a pressure transducer (cat. no. 42584; ICU Medical, Inc., San Clemente, CA, USA) was inserted through the internal jugular vein and threaded to the hepatic vein under guidance from a guide-wire (cat. no. RF-PA3523M; Terumo Corporation, Tokyo, Japan) and intravenous contrast (iopromide injection; Bayer, Shanghai, China). Free hepatic venous pressure (FHVP) and wedged hepatic venous pressure (WHVP) or occluded venous pressure were measured after repeated calibration to ensure accurate measurements. The difference between FHVP and WHVP was calculated and recorded as HVPG.

$C T$. All patients undergoing abdominal contrast CT were required to fast for a minimum of $4 \mathrm{~h}$ prior to the examination. The $5 \mathrm{~mm}$ transverse, sagittal and coronal planes were utilized for the purpose of this study.

Liver volume and spleen volume measurements. A 3D rendering model for liver and spleen were constructed using IQQA-Liver (version 1.2.5; EDDA Technology, Inc., Princeton, NJ, USA) for volume measurements. Pre-contrast, arterial and venous phase abdominal CT images at $5 \mathrm{~mm}$ slices were used for volume rendering. Two authors (YJT and XQZ) manually traced the area of both organs in the sagittal, coronal and transverse planes. IQQA-Liver allows for an automatic construction of a 3D rendering model for the traced region. Each transverse plane was meticulously reviewed and corrected for any inconsistencies. All volume measurements were measured in $\mathrm{cm}^{3}$. A sample of the volume calculation process is presented in Fig. 1.

Clinical data. All laboratory parameters were collected upon hospital admission, including total bilirubin (3.4-20.4 $\mu \mathrm{mol} / \mathrm{l})$, albumin (35-55 g/l), AST (7-40 U/l), ALT (13-35 U/1), serum creatinine (44-115 $\mu \mathrm{mol} / \mathrm{l})$, hemoglobin $(115-150 \mathrm{~g} / \mathrm{l})$, platelet 
Table I. Clinical characteristics of study population $(n=77)$.

\begin{tabular}{|c|c|}
\hline Variables & $\begin{array}{c}\text { Mean } \pm \text { standard } \\
\text { deviation or } \mathrm{n}(\%)\end{array}$ \\
\hline \multicolumn{2}{|l|}{ Sex } \\
\hline Male & $48(62.3)$ \\
\hline Female & $29(37.7)$ \\
\hline Age (years) & $56.78 \pm 12.09$ \\
\hline HVPG (mmHg) & $14.25 \pm 6.18$ \\
\hline \multicolumn{2}{|l|}{ Laboratory findings } \\
\hline Total bilirubin & $18.93 \pm 20.58$ \\
\hline Albumin (g/l) & $34.65 \pm 4.88$ \\
\hline ALT & $27.56 \pm 17.24$ \\
\hline AST & $37.52 \pm 20.63$ \\
\hline Creatinine & $67.18 \pm 15.89$ \\
\hline Hemoglobin & $93.06 \pm 26.51$ \\
\hline Platelet & $75.04 \pm 51.01$ \\
\hline APRI & $1.29 \pm 0.96$ \\
\hline Prothrombin time (sec) & $14.15 \pm 1.48$ \\
\hline \multicolumn{2}{|l|}{ Radiological findings } \\
\hline Liver volume $\left(\mathrm{cm}^{3}\right)$ & $1,138.81 \pm 407.89$ \\
\hline Spleen volume $\left(\mathrm{cm}^{3}\right)$ & $848.73 \pm 399.13$ \\
\hline Liver volume: Spleen volume & $1.61 \pm 0.92$ \\
\hline Spleen volume: Platelet & $15.96 \pm 12.19$ \\
\hline Child Pugh score & $6.26 \pm 1.49$ \\
\hline
\end{tabular}

Child-Pugh class

$\begin{array}{lc}\text { A } & 49(63.6) \\ \text { B } & 26(33.8) \\ \text { C } & 2(2.6) \\ \text { Etiology } & \\ \text { Viral cirrhosis } & 47(61.0) \\ \text { Non-viral cirrhosis } & 30(39.0) \\ \text { GOV type } & \\ \text { GOV1 } & 38(49.4) \\ \text { GOV2 } & 22(28.6) \\ \text { IGV1 } & 8(10.4) \\ \text { IGV2 } & 0(0.0) \\ \text { EV } & 9(11.7) \\ \text { Treatment } & \\ \text { None } & 6(7.8) \\ \text { EBL } & 10(13.0) \\ \text { EIS } & 1(1.3) \\ \text { Cyanoacrylate injection } & 2(2.6) \\ \text { EBL + cyanoacrylate injection } & 46(59.7) \\ \text { BRTO } & 12(15.6) \\ \text { Variceal rebleed } & \\ \text { Rebleed } & 19(24.7) \\ \text { No rebleed } \geq 6 \text { months } & 58(75.3)\end{array}$

HVPG, hepatic venous pressure gradient; ALT, alanine aminotransferase; AST, aspartame aminotransferase; APRI, aminotransferase-to-platelet ratio index; GOV, gastroesophageal varices; IGV, isolated gastric varices; EV, esophageal varices; EIS, endoscopic injection sclerotherapy; EBL, endoscopic band ligation; BRTO, balloon-occluded retrograde transvenous obliteration.
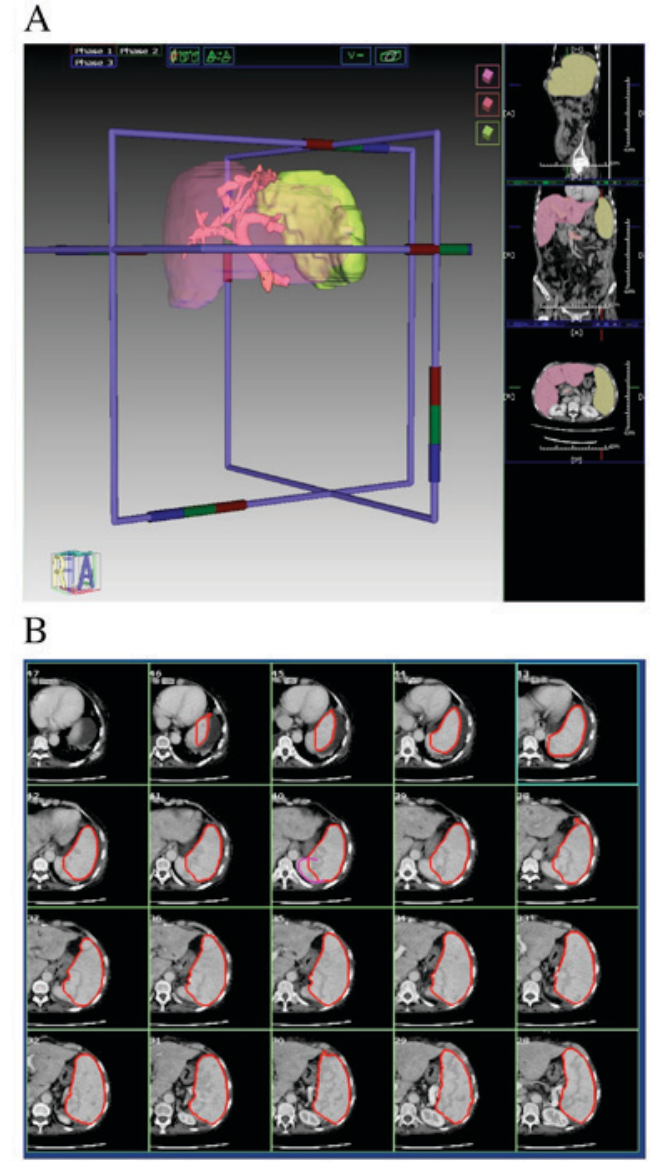

Figure 1. (A) 3D volume rendering with IQQA-Liver provides a precise estimation of spleen and liver volume in patients with portal hypertension. (B) Sequential transverse planes were reviewed and corrected for any inconsistencies.

(125-350x 10 $/ 1)$, prothrombin time (10.0-13.0 sec) and amino transferase to platelet ratio index (APRI). The Child-Pugh Score for each patient were also calculated (14). Patient histories were retrospectively reviewed and assessed for the presence or absence of the following comorbidities: Portal venous thrombosis, hepatocellular carcinoma, spontaneous portosystemic shunt and ascites. Follow-ups were conducted 6 months following the initial endoscopic procedure through telephone interviews or review of readmission medical records. The incidence of variceal rebleed and mortality were recorded.

Statistical analysis. A univariate linear regression analysis was conducted to assess the association between HVPG and an independent continuous variable, including total bilirubin, albumin, ALT, AST, creatine, hemoglobin, platelet, APRI, prothrombin time, age, liver volume, spleen volume, liver to spleen volume, spleen volume to platelet and Child-Pugh score. All variables achieving statistical significance at a 0.20 level were considered in the multivariate linear regression model. A backward variable selection was employed to identify a probable prediction model at the significance level of 0.05 . An ROC curve was used to assess the HVPG predictive model for accurate identification of clinically significant portal hypertension (CSPH or HVPG $\geq 10 \mathrm{mmHg}$ ). Comparisons between continuous data were achieved using individual sample t-tests, while categorical data was compared using Chi-square tests. 


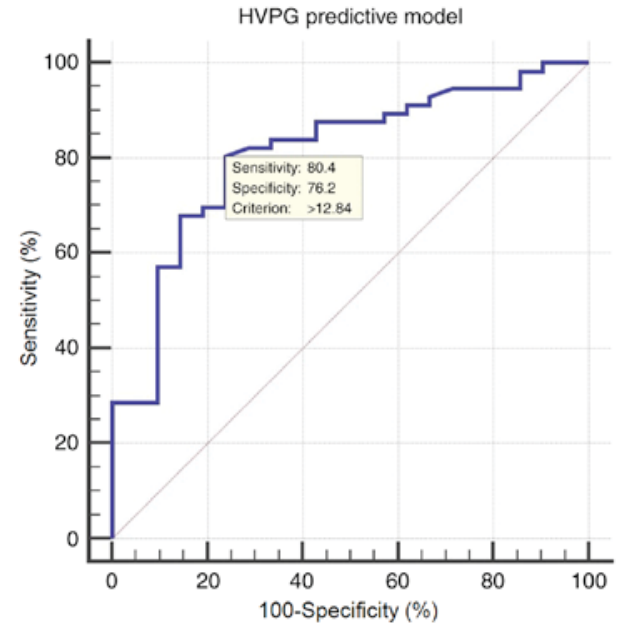

Figure 2. Receiver operating characteristic curve of HVPG predictive model for diagnosing clinically significant portal hypertension, defined as HVPG $\geq 10$ mmHg. HVPG, hepatic vein pressure gradient.

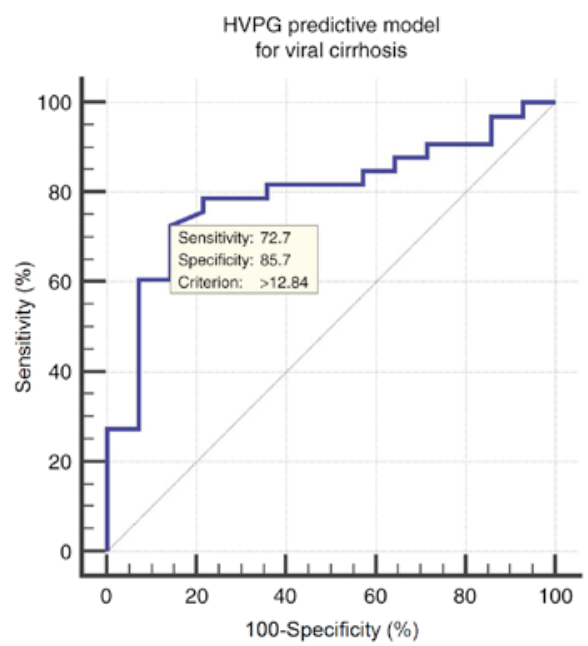

Figure 3. Receiver operating characteristic curve for predicting clinically significant portal hypertension in patients with viral cirrhosis. AUROC of 0.798 (95\% CI, 0.655-0.901), with an optimal cut-off value of 12.84 and a corresponding sensitivity of 72.73 , a specificity of 85.71 . HVPG, hepatic vein pressure gradient. CI, confidence interval.

All statistical analysis was performed using SPSS 22.0 software (IBM Corp., Armonk, NY, USA). P $<0.05$ was considered to indicate a statistically significant difference.

\section{Results}

Baseline characteristics. Of the 77 patients included in the present study, 48 (62.3\%) were male and 29 (37.7\%) were female with a mean age of $56.78 \pm 12.09$ years (range, 24-81 years). The mean HVPG was $14.25 \pm 6.18 \mathrm{mmHg}$ (reference range, $1-5 \mathrm{mmHg}$ ) (15). A total of 49 patients (63.6\%) were Child-Pugh class A, 25 (33.8\%) were class B and 2 (2.6\%) were class $\mathrm{C}$. The mean liver and spleen volumes calculated via $3 \mathrm{D}$ rendering technology were $1,138.81 \pm 407.89$ (reference range, 984-2,439 $\mathrm{cm}^{3}$ ) $(15)$ and $848.73 \pm 399.13 \mathrm{~cm}^{3}$ (reference range, 107.2-314.5 $\left.\mathrm{cm}^{3}\right)(16)$, respectively. Endoscopic examinations revealed the presence of GOV type 1 in 38 patients

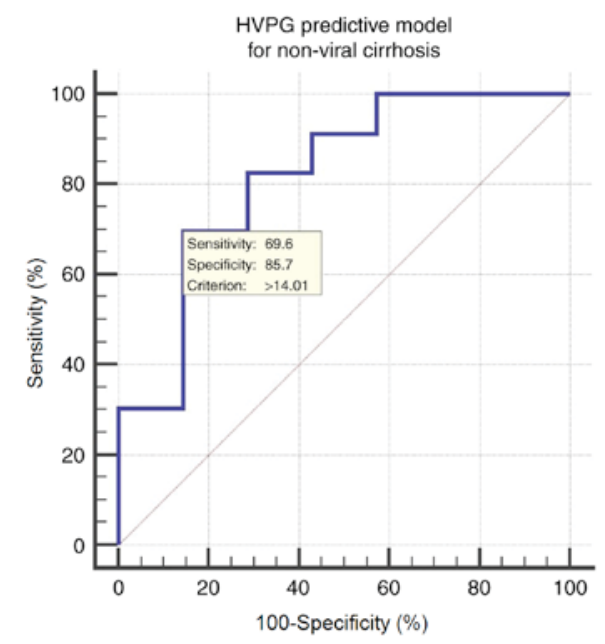

Figure 4. Receiver operating characteristic curve for predicting clinically significant portal hypertension in patients with non-viral cirrhosis. AUROC of 0.820 (95\% CI, 0.637-0.935), with an optimal cut-off value of 14.01 and a sensitivity of 69.57 , a specificity of 85.71 . CI, confidence interval.

(49.4\%), GOV type 2 in 22 patients (28.6\%), IGV type 1 in 8 patients (10.4\%) and esophageal varices (EV) in 9 patients $(11.7 \%)$. No patients included in the present study had IGV type 2. A total of 71 patients received endoscopic therapy as a primary prophylaxis measure, including 10 cases of EBL, 1 case of EIS, 2 cases of cyanoacrylate injection, 46 cases combined EBL + cyanoacrylate injection and 12 cases of BRTO (15-18). The clinical characteristics and laboratory parameters of the study population are presented in Table I.

Non-invasive prediction of HVPG. A univariate linear regression analysis revealed 6 clinically significant continuous variable: Albumin, ALT, AST, APRI, Child-Pugh score, and liver volume. All 6 parameters were entered into a backwards multivariate regression analysis, which resulted in an HVPG predictive model including 3 statistically significant variables: Liver volume, albumin and APRI. The constructed model is as follows: HVPG $=18.726-0.324$ (albumin) +1.57 $(\mathrm{APRI})+0.004$ (liver volume) (multivariate analysis, $\mathrm{P}=0.006$ )

Diagnostic accuracy of the HVPG predictive model. A corresponding ROC curve was constructed for the HVPG predictive model to identify clinically significant portal hypertension, defined as HVPG $\geq 10 \mathrm{mmHg}$. The area under receiver operating characteristic (AUROC) was 0.810 [95\% confidence interval (CI), 0.705-0.891], with an optimal cut-off value of 12.84 yielding a sensitivity of $80.36 \%$, a specificity of $76.19 \%$, a positive predictive value (PPV) of 90.0 and a negative predictive value (NPV) of 59.3 (Fig. 2).

HVPG predictive model for viral vs. non-viral cirrhosis. Of the 77 included study subjects, 47 had viral cirrhosis and 30 patients had non-viral cirrhosis. Viral cirrhosis included 44 cases of hepatitis B and 3 cases of hepatitis C. Non-viral etiologies of cirrhosis included schistosomiasis cirrhosis, alcoholic cirrhosis, primary biliary cirrhosis, and cryptogenic cirrhosis. Details and differences between patients with viral and non-viral cirrhosis are summarized in Table II. 
Table II. Viral cirrhosis vs. non-viral cirrhosis.

\begin{tabular}{|c|c|c|c|}
\hline Variables & Viral cirrhosis $(n=47)$ & Non-viral cirrhosis $(n=30)$ & P-value \\
\hline \multicolumn{4}{|l|}{ Sex } \\
\hline Male & $35(74.5)$ & $13(43.3)$ & \\
\hline Female & $12(25.5)$ & $17(56.7)$ & \\
\hline Age (years) & $55.57 \pm 11.68$ & $58.67 \pm 12.67$ & 0.276 \\
\hline HVPG (mmHg) & $14.06 \pm 6.43$ & $14.53 \pm 5.87$ & 0.747 \\
\hline \multicolumn{4}{|l|}{ Laboratory findings } \\
\hline Total bilirubin & $16.59 \pm 7.24$ & $22.61 \pm 31.69$ & 0.212 \\
\hline Albumin (g/l) & $34.68 \pm 5.39$ & $34.60 \pm 4.04$ & 0.944 \\
\hline ALT & $28.43 \pm 18.33$ & $26.20 \pm 15.56$ & 0.584 \\
\hline AST & $36.0 \pm 20.34$ & $39.90 \pm 21.21$ & 0.422 \\
\hline Creatinine & $71.28 \pm 14.91$ & $60.77 \pm 15.48$ & 0.004 \\
\hline Hemoglobin & $94.79 \pm 26.90$ & $90.37 \pm 26.10$ & 0.479 \\
\hline Platelet & $60.36 \pm 27.51$ & $98.03 \pm 68.71$ & 0.007 \\
\hline APRI & $1.43 \pm 1.08$ & $1.06 \pm 0.69$ & 0.098 \\
\hline Prothrombin time (sec) & $14.26 \pm 1.46$ & $13.99 \pm 1.51$ & 0.451 \\
\hline \multicolumn{4}{|l|}{ Radiological findings } \\
\hline Liver volume $\left(\mathrm{cm}^{3}\right)$ & $1,001.82 \pm 249.34$ & $1,353.42 \pm 509.26$ & 0.001 \\
\hline Spleen volume $\left(\mathrm{cm}^{3}\right)$ & $875.30 \pm 379.16$ & $807.11 \pm 431.87$ & 0.468 \\
\hline Liver volume: Spleen volume & $1.32 \pm 0.58$ & $2.06 \pm 1.16$ & 0.002 \\
\hline Spleen volume: Platelet & $18.59 \pm 12.78$ & $11.84 \pm 10.09$ & 0.017 \\
\hline Child-Pugh score & $6.32 \pm 1.630$ & $6.17 \pm 1.262$ & 0.664 \\
\hline \multicolumn{4}{|l|}{ Child-Pugh class } \\
\hline A & $31(66.0)$ & $18(60.0)$ & \\
\hline $\mathrm{B}$ & $14(29.8)$ & $12(40.0)$ & \\
\hline $\mathrm{C}$ & $2(4.3)$ & $0(0.0)$ & \\
\hline \multicolumn{4}{|l|}{ GOV type } \\
\hline GOV1 & $26(55.3)$ & $12(40.0)$ & \\
\hline GOV2 & $13(27.7)$ & $9(30.0)$ & \\
\hline IGV1 & $5(10.6)$ & $3(10.0)$ & \\
\hline IGV2 & $0(0.0)$ & $0(0.0)$ & \\
\hline EV & $3(6.4)$ & $6(20.0)$ & \\
\hline \multicolumn{4}{|l|}{ Treatment } \\
\hline None & $5(10.6)$ & $1(3.3)$ & \\
\hline EBL & $3(6.4)$ & $7(23.3)$ & \\
\hline EIS & $0(0.0)$ & $1(3.3)$ & \\
\hline Cyanoacrylate injection & $2(4.3)$ & $0(0.0)$ & \\
\hline EBL + Cyanoacrylate injection & $30(63.8)$ & $16(53.3)$ & \\
\hline BRTO & $7(14.9)$ & $5(16.7)$ & \\
\hline \multicolumn{4}{|l|}{ Variceal rebleed } \\
\hline Rebleed & $11(23.4)$ & $8(26.7)$ & \\
\hline No rebleed $\geq 6$ months & $36(76.6)$ & $22(73.3)$ & \\
\hline
\end{tabular}

HVPG, hepatic venous pressure gradient; ALT, alanine aminotransferase; APRI, aminotransferase-to-platelet ratio index; GOV, gastroesophageal varices; IGV, isolated gastric varices; EV, esophageal varices; EIS, endoscopic injection sclerotherapy; EBL, endoscopic band ligation; BRTO, balloon-occluded retrograde transvenous obliteration.

HVPG predictive model in patients with viral cirrhosis. The ROC curve of the HVPG predictive model for determining CSPH (HVPG $\geq 10 \mathrm{mmHg}$ ) in patients with viral cirrhosis resulted in an AUROC of 0.798 (95\% CI; 0.655-0.901). The optimal cut-off value is 12.84 , with a corresponding sensitivity of $72.73 \%$, specificity of $85.71 \%$, a PPV of 92.3 and a NPV of 57.1 (Fig. 3).

HVPG predictive model in patients with non-viral cirrhosis. The ROC curve of the HVPG predictive model constructed 
to determine CSPH (HVPG $\geq 10 \mathrm{mmHg}$ ) in patients with non-viral cirrhosis yielded an AUROC of 0.820 (95\% CI; $0.637-0.935)$. The optimal cut-off value is 14.01 , with a sensitivity of $69.57 \%$, specificity of $85.71 \%$, a PPV of 94.1 and an NPV of 46.2 (Fig. 4).

\section{Discussion}

The aim of the present study was to investigate the potential of liver and spleen volume as a non-invasive predictor for assessing HVPG and gastroesophageal varices in patients with portal hypertension. All patients underwent an upper abdominal CT examination with contrast, transjugular measurement of HVPG, an endoscopic examination to identify the presence of gastroesophageal varices and endoscopic treatment when deemed necessary.

A univariate analysis of all non-invasive parameters identified six clinically significant continuous variables that were entered into a multivariate regression analysis, with a resulting HVPG predictive model: $\mathrm{HVPG}=18.726-0.324$ (albumin) +1.57 (APRI) + 0.004 (liver volume). In order to further understand the suitability of the HVPG predictive model based on clinical etiology, the study subjects were divided into viral $(n=47)$ and non-viral cirrhosis $(n=30)$. Studies revealed that patients with viral cirrhosis tend to have more prominent liver atrophy, whereas liver hypertrophy may be observed in non-viral etiologies, including alcoholic cirrhosis (19). Although the performance of AUROC is slightly better in the non-viral cirrhosis group compared with the viral cirrhosis group, the cut-off value yielded a more desirable diagnostic accuracy for patients with viral cirrhosis. The HVPG predictive model was constructed based on the whole population with varying etiologies, and so the model is feasible for both viral and non-viral cirrhosis.

Previous studies have demonstrated that liver volume to spleen volume ratio may be useful for predicting HVPG score in patients with HVPG $>12 \mathrm{mmHg}(20)$. However, such prediction requires prior knowledge of gastroesophageal variceal classification, which is only possible via an endoscopy exam. To the best of our knowledge, no studies have presented a truly non-invasive diagnostic method for HVPG and gastroesophageal varices in patients with portal hypertension based on liver and spleen volume alone. The early detection of CSPH is important due to its association with cirrhotic complications, including gastroesophageal variceal hemorrhage $(21,22)$. At present, the gold standard for stratifying the degree of portal hypertension is invasive measurement of HVPG (23-25); no effective non-invasive replacement for HVPG measurements is currently available $(6,7,21)$. Various non-invasive methods have been suggested to replace HVPG measurements, ranging from serum biomarkers to imaging modalities or the combination of different techniques $(5,8,26)$. Transient elastography (TE) has yielded the most promising results, however the accuracy of TE may be influenced by comorbidities, including the presence of ascites and obesity $(20,27-30)$. 3D rendering of liver volume based on $\mathrm{CT}$ images has potential for predicting HVPG and assessing disease severity. The widespread availability of CT imaging may allow physicians to accurately assess the full scope of the disease and perhaps monitor treatment responses and disease progression.
There are several limitations to the present study. Due to the limited number of study subjects, a control group was not employed to verify the proposed model. The majority of patients did not undergo a follow-up CT examination following treatment. The utility of the proposed model as a monitor for disease progression remains to be determined. Furthermore, the proposed equation had a positive coefficient for the liver variable, which is uncommon as cirrhosis is typically associated with a decrease in liver volume. A potential explanation for this is the difference in cirrhosis etiologies. Kim et al (19) demonstrated that there is a significant difference in liver volume in patients with cirrhosis of different etiologies. Patients with alcoholic cirrhosis had an average volume of $1,204.2 \mathrm{~cm}^{3}$, whereas those with HBV had a mean volume of $946.6 \mathrm{~cm}^{3}$ (19). In the present study, patients with different etiologies, including viral hepatitis, cirrhosis, primary biliary cirrhosis and alcoholic cirrhosis, were included, which may result in inconsistencies in liver size. Another possible explanation is that body mass index (BMI) may affect liver and spleen size in each individual patient. Unfortunately, BMI data was not available in the present study. In order to confirm the efficacy of the method of HVPG prediction presented in the present study, further, multi-center studies with a larger study population should be performed.

\section{Acknowledgements}

The present study was supported by the Innovation Fund of Shanghai Scientific Committee (grant no. 15411950501) and the Foundation of Shanghai Municipal Commission of Health and Family Planning (grant no. 20154Y0065).

\section{References}

1. Mokdad AA, Lopez AD, Shahraz S, Lozano R, Mokdad AH, Stanaway J, Murray CJ and Naghavi M: Liver cirrhosis mortality in 187 countries between 1980 and 2010: A systematic analysis. BMC Med 12: 145, 2014.

2. Triantos CK, Nikolopoulou V and Burroughs AK: Review article: The therapeutic and prognostic benefit of portal pressure reduction in cirrhosis. Aliment Pharmacol Ther 28: 943-952, 2008.

3. Garcia-Tsao G, Sanyal AJ, Grace ND and Carey W; Practice Guidelines Committee of the American Association for the Study of Liver Diseases; Practice Parameters Committee of the American College of Gastroenterology: Prevention and management of gastroesophageal varices and variceal hemorrhage in cirrhosis. Hepatology 46: 922-938, 2007.

4. Garcia-Tsao G and Bosch J: Management of varices and variceal hemorrhage in cirrhosis. N Engl J Med 362: 823-832, 2010.

5. Procopet B, Cristea VM, Robic MA, Grigorescu M, Agachi PS, Metivier S, Peron JM, Selves J, Stefanescu H, Berzigotti A, et al: Serum tests, liver stiffness and artificial neural networks for diagnosing cirrhosis and portal hypertension. Dig Liver Dis 47: 411-416, 2015.

6. Suk KT: Hepatic venous pressure gradient: Clinical use in chronic liver disease. Clin Mol Hepatol 20: 6-14, 2014.

7. de Franchis R: Baveno VI Faculty: Expanding consensus in portal hypertension: Report of the Baveno VI consensus workshop: Stratifying risk and individualizing care for portal hypertension. J Hepatol 63: 743-752, 2015.

8. Procopet B, Berzigotti A, Abraldes JG, Turon F, Hernandez-Gea V, Garcia-Pagan JC and Bosch J: Real-time shear-wave elastography: Applicability, reliability and accuracy for clinically significant portal hypertension. J Hepatol 62: 1068-1075, 2015.

9. Merkel C and Montagnese S: Should we routinely measure portal pressure in patients with cirrhosis, using hepatic venous pressure gradient (HVPG) as guidance for prophylaxis and treatment of bleeding and re-bleeding? Yes! Eur J Intern Med 22: 1-4, 2011. 
10. Ferenci P, Lockwood A, Mullen K, Tarter R, Weissenborn K and Blei AT: Hepatic encephalopathy-definition, nomenclature, diagnosis and quantification: Final report of the working party at the 11th World Congresses of Gastroenterology, Vienna, 1998. Hepatology 35: 716-721, 2002.

11. Bedossa P and Poynard T: An algorithm for the grading of activity in chronic hepatitis C. The METAVIR Cooperative Study Group Hepatology 24: 289-293, 1996.

12. Sarin SK, Lahoti D, Saxena SP, Murthy NS and Makwana UK: Prevalence, classification and natural history of gastric varices: A long-term follow-up study in 568 portal hypertension patients. Hepatology 16: 1343-1349, 1992.

13. Sarin SK and Lahoti D: Management of gastric varices. Baillieres Clin Gastroenterol 6: 527-548, 1992

14. Cholongitas E, Papatheodoridis GV, Vangeli M, Terreni N, Patch D and Burroughs AK: Systematic review: The model for end-stage liver disease-should it replace Child-Pugh's classification for assessing prognosis in cirrhosis? Aliment Pharmacol Ther 22:1079-1089, 2005.

15. Sarin SK and Kumar A: Endoscopic treatment of gastric varices. Clin Liver Dis 18: 809-827, 2014.

16. Sarin SK: Injection sclerotherapy for varices. Trop Gastroenterol 8: 177-181, 1987

17. Saad WE: Balloon-occluded retrograde transvenous obliteration of gastric varices: Concept, basic techniques, and outcomes. Semin Intervent Radiol 29: 118-128, 2012.

18. Garcia-Pagán JC, Barrufet M, Cardenas A and Escorsell À: Management of gastric varices. Clin Gastroenterol Hepatol 12: 919-928, 2014

19. Kim I, Jang YJ, Ryeom H, Lee SM, Lee HJ, Kim GC and Kim HJ: Variation in hepatic segmental volume distribution according to different causes of liver cirrhosis: CT volumetric evaluation. J Comput Assist Tomogr 36: 220-225, 2012.

20. Yan S, Wu H, Wang G, Chen Y, Zhang C and Zhu Q: A new model combining the liver/spleen volume ratio and classification of varices predicts HVPG in hepatitis B patients with cirrhosis. Eur J Gastroen Hepat 27: 335-343, 2015.

21. de Franchis R; Baveno V Faculty: Revising consensus in portal hypertension: report of the Baveno $\mathrm{V}$ consensus workshop on methodology of diagnosis and therapy in portal hypertension. J Hepatol 53: 762-768, 2010.

22. Kim TY, Jeong WK, Sohn JH, Kim J, Kim MY and Kim Y: Evaluation of portal hypertension by real-time shear wave elastography in cirrhotic patients. Liver Int 35: 2416-2424, 2015.
23. Kim MY, Jeong WK and Baik SK: Invasive and non-invasive diagnosis of cirrhosis and portal hypertension. World J Gastroenterol 20: 4300-4315, 2014.

24. Abraldes JG, Sarlieve P and Tandon P: Measurement of portal pressure. Clin Liver Dis 18: 779-792, 2014.

25. Silva-Junior G, Baiges A, Turon F, Torres F, Hernández-Gea V, Bosch J and Garcia-Pagan JC: The prognostic value of hepatic venous pressure gradient in patients with cirrhosis is highly dependent on the accuracy of the technique. Hepatology 62: 1584-1592, 2015

26. Berzigotti A, Reverter E, García-Criado A, Abraldes JG, Cerini F. García-Pagán JC and Bosch J: Reliability of the estimation of total hepatic blood flow by Doppler ultrasound in patients with cirrhotic portal hypertension. J Hepatol 59: 717-722, 2013.

27. Lemoine M, Katsahian S, Ziol M, Nahon P, Ganne-Carrie N, Kazemi F, Grando-Lemaire V, Trinchet JC and Beaugrand M: Liver stiffness measurement as a predictive tool of clinically significant portal hypertension in patients with compensated hepatitis $\mathrm{C}$ virus or alcohol-related cirrhosis. Aliment Pharm Ther 28: 1102-1110, 2008.

28. Hong WK, Kim MY, Baik SK, Shin SY, Kim JM, Kang YS, Lim YL, Kim YJ, Cho YZ, Hwang HW, et al: The usefulness of non-invasive liver stiffness measurements in predicting clinically significant portal hypertension in cirrhotic patients: Korean data. Clin Mol Hepatol 19: 370-375, 2013

29. Zhang QW, Wang Y, Wang J, Zhao HB, Yu H, Liu SY, Zeng X, Chen Q, Hu ZQ, Guo WY, et al: A non-invasive magnetic resonance imaging-based model predicts portal venous pressure. J Digest Dis 17: 175-185, 2016.

30. Hayashi H, Beppu T, Okabe H, Nitta H, Imai K, Doi K, Chikamoto A and Baba H: Combined measurements of serum bile acid level and splenic volume may be useful to noninvasively assess portal venous pressure. J Gastroenterol 47: 1336-1341, 2012.

(i) $($ ) This work is licensed under a Creative Commons Attribution-NonCommercial-NoDerivatives 4.0 International (CC BY-NC-ND 4.0) License. 